\title{
AUTOMORPHISM GROUPS OF PARTIAL ORDERS
}

BY ELLIOTT H. BIRD

Communicated by Gian-Carlo Rota, February 5, 1973

1. The automorphism group $\Gamma(P)$ of a partial order $P$ is the collection of all order preserving permutations (automorphisms) of $P$, a subgroup of the symmetric group on $P$. If $P$ and $Q$ are partial orders then $P \times Q$ becomes a partial order by reverse lexicography: $(p, q)<\left(p^{\prime}, q^{\prime}\right)$ if $q<q^{\prime}$ or $q=q^{\prime}$ and $p<p^{\prime}$. If $f$ is a function whose domain contains the element $a$, we use af to denote the image of $a$ under $f$.

THEOREM 1. $\Gamma(P \times Q)$ contains an isomorphic copy of $\Gamma(P)$ wr $\Gamma(Q), a$ nonstandard wreath product of $\Gamma(P)$ by $\Gamma(Q)$.

Proof. Let $(b, f)$ be an element of $\Gamma(P)$ wr $\Gamma(Q)$, with $f: Q \rightarrow \Gamma(P)$ and $b \in \Gamma(Q)$, where $\Gamma(Q)$ may be identified with a subgroup of $\operatorname{Aut}\left(\prod_{q \in Q} \Gamma(P)\right)$. The action of $b$ on $f$ is defined by $q(f b)=\left(q b^{-1}\right) f$. Define

by

$$
\phi: \Gamma(P) \text { wr } \Gamma(Q) \rightarrow \Gamma(P \times Q)
$$

$$
(p, q)[(b, f) \phi]=(p[q b f], q b) .
$$

It is not difficult to show that $\phi$ is an embedding.

Definition. $\Gamma(P \times Q)$ is $\beta$-imprimitive if the sets $P \times\{q\}, q \in Q$, are sets of imprimitivity (i.e. if for all $\alpha \in \Gamma(P \times Q),\left(p_{1}, q\right) \alpha=\left(p_{1}^{\prime}, q^{\prime}\right)$ and $\left(p_{2}, q\right) \alpha=\left(p_{2}^{\prime}, q^{\prime \prime}\right)$ implies $\left.q^{\prime}=q^{\prime \prime}\right)$.

The author wishes to thank Jack Sonn for simplifying the proof of the sufficiency of the following theorem.

THEOREM 2. $\Gamma(P \times Q) \cong \Gamma(P)$ wr $\Gamma(Q)$ if and only if $\Gamma(P \times Q)$ is $\beta$-imprimitive.

Proof. If $\phi$ is an isomorphism then every $\alpha \in \Gamma(P \times Q)$ behaves algebraically like one $(b, f) \in \Gamma(P)$ wr $\Gamma(Q)$, and $\beta$-imprimitivity follows from the definition of $(b, f)$. Conversely, if $\Gamma(P \times Q)$ is $\beta$-imprimitive, then each $\alpha \in \Gamma(P \times Q)$ induces an $\alpha^{*} \in \Gamma(Q)$ and the mapping $\alpha \rightarrow \alpha^{*}$ is an epimorphism. It follows that the diagram

$$
\begin{array}{ccc}
1 \rightarrow \Gamma(P) & \rightarrow \Gamma(P \times Q) & \rightarrow \Gamma(Q) \rightarrow 1 \\
\| & \phi \uparrow & \| \\
1 & \rightarrow \Gamma(P) \rightarrow \Gamma(P) \text { wr } \Gamma(Q) \rightarrow \Gamma(Q) \rightarrow 1
\end{array}
$$

AMS (MOS) subject classifications (1970). Primary 06A10; Secondary 05C25. 
is commutative with exact rows and, by the five lemma, $\phi$ is an isomorphism.

THEOREM 3. If $P$ is a partial order and $P^{\circ}$ is the partial order obtained by adjoining a universal lower bound to $P$, then $\Gamma\left(P^{\circ}\right) \cong \Gamma(P)$.

THEOREM 4. If $P$ is a finite partial order with universal lower bound and $Q$ is a finite partial order, then $\Gamma(P \times Q)$ is $\beta$-imprimitive.

The proof is by induction on $h(q), h$ being the height function on $Q: h(q)=\sup \left\{\right.$ lengths of maximal subchains from $q_{0}$ to $\left.q\right\}$, where the supremum is taken over all minimal elements $q_{0}$ in $Q$, for which $q_{0} \leqq q$.

Theorem 4 tells us that, at least in the finite case, we can be certain that the automorphism group on $P \times Q$ is the wreath product $\Gamma(P)$ wr $\Gamma(Q)$ if only $P$ has a universal lower bound, and if $P$ does not, then by adjunction of a lower bound, that wreath product is obtained (Theorem 3). Details of the proofs may be found in [1] or [2].

2. Having obtained a wreath product it is natural to ask whether the standard wreath product can be obtained (as an automorphism group of a partial order). Given an arbitrary group $A$ with a well-ordered generating system $\left\{a_{j} ; j \in J\right\}$, Frucht [5] has shown there is a partial order $\Phi_{A}$ for which $\Gamma\left(\Phi_{A}\right) \cong A$. Namely $\Phi_{A}=A \times(2+J)$ with

$$
\begin{array}{clrl}
(a, i) & <(a, j), & a \in A & \text { and } i<j<2+J, \\
\left(a_{i} a, 1\right) & <(a, 2+j), & a \in A & \text { and } i \leqq j<2+J .
\end{array}
$$

Using Frucht orders $\Phi_{A}, \Phi_{B}$ for two groups $A$ and $B$, with generating systems of order types $J, I$, respectively, we next construct a partial order whose automorphism group is the standard wreath product $A \backslash B$, and which is more economical than the Frucht order for $A\} B$.

Definition. A partial order $P$ is called uniform if (i) every subset of $P$ has minimal elements and (ii) if $p, p^{\prime} \in P$ such that $h(p)=h\left(p^{\prime}\right)$, where $h$ is the height function, then $p$ and $p^{\prime}$ are in the same orbit of $\Gamma(P)$.

Note that the orbits of $\Gamma(P)$ are well-ordered; we denote the minimal orbit by $\Theta_{P}$, or if no ambiguity can arise, by $\Theta$. As an important example, Frucht orders are uniform.

Definition. If $P$ and $Q$ are uniform partial orders, we define

$$
P\left\{Q=\left(P \times \Theta_{Q}\right) \cup\left(Q-\Theta_{Q}\right),\right.
$$

with the order in $P \times \Theta_{Q}$ determined by that of $P$, the order in $Q-\Theta_{Q}$ remaining unchanged, and $(p, r)<q$ if $r<q$ in $Q$.

While $P\{Q$ is a uniform partial order, it is not in general true that $\Gamma(P \mid Q)$ is a wreath product. However, if $P$ is a Frucht order, we do obtain a wreath product, and if $Q$, too, is a Frucht order, a standard wreath 
product is obtained. We prove the latter first.

TheOREM 5. $\left.\left.\Gamma\left(\Phi_{A}\right\} \Phi_{B}\right) \cong A\right\} B$.

Proof. Major steps in the proof are listed here. We use $\lambda$ generically to denote an element of $W=\Gamma\left(\Phi_{A} \backslash \Phi_{B}\right)$. Note that a typical element of $\Phi_{A} \backslash \Phi_{B}$ has the form $((a, j),(b, 1))$, where $j<J, a \in A, b \in B$, if it belongs to $\Phi_{A} \times \Theta_{B}$ and the form $(b, i)$, where $b \in B$ and $1<i<I$ if it belongs to $\Phi_{B}-\Theta_{B}$, and where $\Theta_{B}=B \times\{1\}$ is the minimal orbit of $\Phi_{B}$.

(i) If $((e, 1),(b, 1)) \lambda=\left((a, 1),\left(b^{\prime}, 1\right)\right)$, where $e=e_{A}$ is the identity of $A$, $a$ is in $A, b$ and $b^{\prime}$ in $B$, then $((g, j),(b, 1)) \lambda=\left((g a, j),\left(b^{\prime}, 1\right)\right)$ for all $j<2+J$ and all $g$ in $A$, and $(b, i) \lambda=\left(b^{\prime}, i\right)$ for all $i$ with $1<i<2+I$.

(ii) $K=\left\{k \in W ;\left(k \mid \Phi_{A} \times \Theta_{B}\right) \pi_{1}=\pi_{1}\right\}$, where $\pi_{1}$ is the projection on the first component, is a subgroup of $W$ isomorphic to $B$.

(iii) $F=\left\{f \in W ;\left(f \mid \Phi_{A} \times \Theta_{B}\right) \pi_{2}=\pi_{2}\right\}$ is a normal subgroup of $W$ isomorphic to $\prod_{b \in B} A_{b}$, where $A_{b} \cong A$ for each $b$ in $B$.

(iv) $W$ is a semidirect product of $F$ by $K$, and so is (isomorphic to) a semidirect product of $\prod_{b} A$ and $B$.

(v) The mapping $\alpha: K \rightarrow \operatorname{Aut}(F)$ defined by $f(k \alpha)=k^{-1} f k$ is an embedding.

Hence $W=\Gamma\left(\Phi_{A} \backslash \Phi_{B}\right)$ is the relative holomorph of $\prod_{b} A$ by $B$, i.e. the standard wreath product of $A$ by $B$. We point out that the groups $\Gamma\left(\Phi_{A} \backslash \Phi_{B}\right)$ and $A \nmid B$ are not isomorphic as permutation groups, but only as groups.

Corollary. For $\left.\lambda \in \Gamma\left(\Phi_{A}\right\} \Phi_{B}\right)$, $\lambda$ induces a map $\lambda^{*} \in \Gamma\left(\Phi_{B}\right)$ such that $\lambda^{*}\left|\Phi_{B}-\Theta_{B}=\lambda\right| \Phi_{B}-\Theta_{B}$. The mapping $\lambda \rightarrow \lambda^{*}$ is an epimorphism.

The proof of the next theorem mimics that of the preceding one. Here only one of the uniform partial orders is a Frucht representation.

THEOREM 6. Let $Q$ be a uniform partial order. Let $A$ be a group with Frucht representation $\Phi_{A}$. Then the automorphism group of $\Phi_{A} \backslash Q$ is a wreath product (in general, nonstandard) of $A$ by $\Gamma(Q)$.

Observe that $\Gamma\left(\Phi_{A} \backslash Q\right)$ is a standard wreath product if and only if $\Gamma(Q)$ is isomorphic as a permutation group on $\Theta_{Q}$ to a Cayley representation. Details of the proofs of Theorems 5 and 6 may be found in [1] or [3].

3. Definition. Let $A$ be a group and $P$ a uniform partial order with minimal orbit $\Theta$ such that $\Gamma(P) \cong A$. A group $B$ is said to be obtainable from $A$ if there exist a nonempty set $S$ and a surjection $f: \Theta \rightarrow S$ such that if $Q=P \cup S$ with $p>s$ if and only if $f(p)=s$, for $p$ in $P$ and $s$ in $S$, then $\Gamma(Q) \cong B$.

What is being done here is that a new family of minimal orbits is adjoined to a uniform partial order with each member of the original 
minimal orbit having a unique predecessor. (Note that the resulting order need not be uniform.) We will write the image of $p \operatorname{in} \Theta$ under $f$ as $f(p)$ contrary to our usual notation.

THEOREM 7. If $B$ is obtainable from $A$, then $B$ is embedded as a subgroup of $A$ and if $|A| \neq 2$, then every subgroup of $A$ is obtainable from $A$.

Proof. If $B$ is obtainable from $A$, then identifying $A$ with $\Gamma(P)$ and $B$ with $\Gamma(Q)$, the mapping $\psi: B \rightarrow A$ defined by $b \psi=b \mid P$, the restriction of $b$ to $P$, is an embedding.

Conversely, let $B$ be a subgroup of $A$ and $\Phi_{A}$ the Frucht representation of $A$. Let $S=\{A-B\} \cup B$; i.e. the elements of $S$ are the points of $B$ and the set $A-B$. Define $f: \mathrm{A} \times\{1\} \rightarrow S$ by $f(\mathrm{a}, 1)=a$ if $a \in B$, and $f(a, 1)=A-B$ if $a \notin B$. If $Q=\Phi_{A} \cup S$ with $(a, 1)>f(a, 1)$, then $\Gamma(Q) \cong B$. In particular, $\psi: \Gamma(Q) \rightarrow A$ defined as above is an isomorphism of $\Gamma(Q)$ onto $B \subseteq A$.

4. A theorem of Kaloujnine and Krasner [6] states that every extension of $A$ by $B$ can be found embedded in $A\} B$. Using Theorems 5 and 6 we indicate here a natural way of producing partial order representations for those extensions which are split, i.e. for the semidirect products of $A$ by $B$.

Specifically, if $\left.\Phi_{A}\right\rceil \Phi_{B}$ is the wreath product representation of $\left.A\right\} B$ and $D=B A$ is a semidirect product of $A$ by $B$ with homomorphism $\alpha$, we let $S=A$ and $f: \Theta \rightarrow S$ by $f((a, 1),(b, 1))=a(b \alpha)$, where

$$
\Theta=(A \times\{1\}) \times(B \times\{1\})
$$

is the minimal orbit of $\left.\Phi_{A}\right\} \Phi_{B}$. We have $\alpha: B \rightarrow \operatorname{Aut}(A)$, and $S$ and $f$ define a new partial order $Q$ as in the definition of "obtainable". From Theorem 6, the automorphism group of $Q$ is (isomorphic to) a subgroup of the automorphism group of $\left.\Phi_{A}\right\rceil \Phi_{B}$, that is a subgroup of $A \backslash B$. We show next that the new automorphism group is isomorphic to $D$.

THEOREM 8. Let $D$ be a semidirect product of $A$ by $B$ with homomorphism $\alpha$. Let $\Phi_{A}$ and $\Phi_{B}$ be the Frucht orders of $A$ and $B$ respectively. Let $Q=\Phi_{A} \nmid \Phi_{B} \cup A$ with $((a, 1),(b, 1))>a(b \alpha)$. Then $\Gamma=\Gamma(Q) \cong D$.

The proof is accomplished in a series of steps showing that (1) $\Gamma$ is (isomorphic to) a subgroup of $A \int B$; (2) for $\rho \in \Gamma$, if $\left(\left(e_{A}, 1\right),\left(e_{B}, 1\right)\right) \rho=$ $((y, 1),(z, 1))$, where $e_{A}, e_{B}$ are the identities of $A, B$ respectively, $y \in A$, $z \in B$, and if $a_{0}=y(z \alpha)$, then $e_{A} \rho=y(z \alpha)=a_{0}$ and $a \rho=a(z \alpha) a_{0}$ for every $a$ in $A$; (3) if $((a, 1),(b, 1)) \rho=\left(\left(a^{\prime}, 1\right),\left(b^{\prime}, 1\right)\right)$, where $a, a^{\prime}$ are in $A$ and $b, b^{\prime}$ are in $B$, then $a^{\prime}=a\left[a_{0}\left(b^{\prime-1} \alpha\right)\right]$ and $\left(b^{-1} b^{\prime}\right) \alpha=z \alpha ;(4) A$ is embedded as a normal subgroup of $\Gamma$ and $B$ as a subgroup of $\Gamma$; and finally (5) $\Gamma \cong B A=D$. 
5. Having constructed a partial order $Q$ whose automorphism group is the semidirect product of $A$ by $B$, we next consider the behavior of the automorphism group of $Q$ when we modify $Q$ by procedures similar to those used in constructing $Q$ itself. Details of the proofs of the following theorem and the preceding one may be found in [1] or [4].

THEOREM 9. Let $D$ be a semidirect product of $A$ by $B$ with homomorphism $\alpha(\alpha: B \rightarrow \operatorname{Aut}(A))$, and let $Q$ be the partial order representation of $D$ constructed in Theorem 8 . Let $C$ be a group with Frucht representation $\Phi_{C}$, $\beta: A \rightarrow \operatorname{Aut}(C)$ a homomorphism, and $P=C \cup \Phi_{C} \backslash Q$ with $((c, 1), a)>$ $c(a \beta)$. Then if $\Gamma=\Gamma(P)$, we have $\Gamma \cong \bar{B} A C$, where $\bar{B}=\{b \in B ;(b \alpha) \beta=\beta\}$ is a subgroup of $B$ with $\left(b_{1} a_{1} c_{1}\right)\left(b_{2} a_{2} c_{2}\right)$ given by

$$
\left(b_{1} a_{1} c_{1}\right)\left(b_{2} a_{2} c_{2}\right)=b_{1} b_{2}\left(a_{1}\left(b_{2} \alpha\right)\right) a_{2}\left(c_{1}\left(a_{2} \beta\right)\right) c_{2} .
$$

6. Acknowledgement. The author wishes to thank David Lubell for guidance in the developments of the contents of this paper.

\section{REFERENCES}

1. E. H. Bird, Automorphism groups of partial orders, Ph.D. Dissertation, Adelphi University, Garden City, N.Y., 1972.

2. - The automorphism group of a product of partial orders, Discrete Math. (to appear).

3. - A partial order representation of the standard wreath product, Discrete Math. (to appear).

4. - A partial order representation of the semi-direct product, Discrete Math. (to appear).

5. R. Frucht, On the construction of partially ordered systems with a given group of automorphisms, Amer. J. Math. 72 (1950), 195-199. MR 11, 320.

6. M. Krasner and L. Kaloujnine, Produit complet des groupes de permutations et le problème d'extension des groupes. III, Acta Sci. Math. Szeged. 14 (1951), 69-82. MR 14, 242.

Department of Mathematics, Long Island University, C. W. Post College, GreEnVAle, New York 11548 